\title{
Self-Stabilizing construction of Bounded Size Clusters
}

\author{
Colette Johnen \\ LRI, Univ. Paris-Sud, CNRS \\ F-91405 Orsay Cedex, France \\ colette@1ri.fr
}

\author{
Le Huy Nguyen \\ LRI, Univ. Paris-Sud, CNRS \\ F-91405 Orsay Cedex, France \\ lehuy@1ri.fr
}

\begin{abstract}
Clustering means partitioning nodes into groups called clusters, providing the network with a hierarchical organization.

A self-stabilizing protocol, regardless of the initial system state, automatically converges to a set of states that satisfy the problem specification without external intervention. Due to this property, self-stabilizing protocols are adapted to highly dynamic networks as ad hoc or sensors networks. In this paper, we propose a self-stabilizing clustering protocol. Our protocol guarantees a threshold (SizeBound) on the number of nodes that a clusterhead handle. Therefore, none of the clusterheads are overloaded at any time. The criterion of the clusterheads election is based on their weight value, a general parameter that can be computed according to several node parameters as transmission power, battery power, ....
\end{abstract}

\section{Introduction}

An ad hoc network is a self-organized network, especially those with wireless or temporary plug-in connections. Ad hoc networks cannot rely on centralized and organized network management. Significant examples include establishing survivable, efficient, dynamic communication for emergency/rescue operations, disaster relief efforts, and military networks. Meetings where participants create a temporary wireless ad hoc network is another typical example. In these networks, mobile nodes may move arbitrary often; thus, the network's topology may change rapidly and unpredictably. Self-configuring, self-healing and self-organizing management is needed in such networks.

Clustering means partitioning network nodes into groups called clusters, providing the network with a hierarchical organization. A cluster is a connected subgraph of the global networks composed of a clusterhead and ordinary nodes. Each node belongs to only one cluster. In addition, a cluster is required to obey to certain constraints that are used for network management, routing methods, resource allocation, etc. By dividing the network into non-overlapped clusters, intra-cluster routing is administered by the clusterhead and inter-cluster routing can be achieved in a reactive manner between clusterheads. It is experimentally shown that these protocols, when coupled with an ad hoc routing protocol, produce throughput improvements of up to $80 \%$ over the ad hoc routing protocol alone [6]. Members of a cluster can share resources such as software, memory space, printer, etc. Also, clustering facilitates the reuse of resources, which improves the system capacity. Moreover, clustering can be used to reduce the amount of information that is used to store the network state. Distant nodes outside of a cluster usually do not need to know the detailed state this cluster. Indeed, an overview of the cluster's state is generally sufficient for those distant nodes to make control decisions. Thus, the clusterhead is typically in charge of collecting the state of nodes in its cluster and constructing an overview of its cluster state.

For the above mentioned reasons, it is not surprising that several distributed clustering protocols have been proposed during the last ten years $[2,3,4,7,9,17]$. A detailed survey on clustering algorithms can be found in [17]. The clustering protocols in $[2,9]$ construct a spanning tree. Then the clusters are constructed on top of the spanning tree. The clusterheads set does not necessarily form a dominating set (i.e., a node can be at distance greater than 1 from its clusterhead). In [17], the performance of the node-degree based clustering algorithms are evaluated. In [7], the weightbased distributed clustering protocol taking into account several parameters (node's degree, transmission and battery power, node mobility) is presented. In a neighborhood, the selected nodes are those that are the most suitable for the clusterhead role (i.e., a node optimizing all the parameters). In [4], a Distributed and Mobility-Adaptive Clustering protocol, called DMAC, is presented. The clusterheads are selected according to a node's parameter (called weight). The higher is the weight of a node, the more suitable this node is for the role of clusterhead. An extended version of this protocol, called Generalized DMAC (GDMAC), is proposed 
in [3].

A system is self-stabilizing [8] when regardless of its initial configuration, it is guaranteed to reach a legitimate configuration in a finite number of steps. A system which is not self-stabilizing may stay in an illegitimate configuration forever. The correctness of self-stabilizing protocols does not depend on initialization of variables, and a self-stabilizing protocol converges to some predefined stable configuration starting from an arbitrary initial one. Selfstabilizing protocols are thus, inherently tolerant to transient faults in the system. Many self-stabilizing protocols can also adapt dynamically to changes in the network topology or system parameters (e.g., communication speed, number of nodes). A new configuration resulting from a topological changes is viewed as an inconsistent configuration from which the system will converge to a configuration consistent with the new topology. Several self-stabilizing protocols for cluster formation and clusterhead selection have been proposed [5, 11, 12, 14, 16]. [14] presents a robust selfstabilizing version of DMAC under the synchronous schedule. A self-stabilizing version of DMAC and GDMAC is presented in [12]. A robust and self-stabilizing version of GDMAC is presented in [11]. In [5], a self-stabilizing linkcluster protocol under an asynchronous message-passing system model is presented. The definition of cluster is not exactly the same as ours: an ordinary node can be at distance two of its clusterhead. The presented clustering protocol requires three types of messages, our protocol adapted to message passing model requires one type of messages. A self-stabilizing protocol for cluster formation is presented in [16]. A density criteria (defined in [15]) is used to select clusterhead: a node $v$ chooses in its neighborhood the node having the biggest density. A $v$ 's neighborhood contains all nodes at distance less or equal to 2 from $v$. Therefore, to choose clusterhead, communication at distance 2 is required. Our protocol builds clusters on local information; thus, it requires only communication between nodes at distance 1 of each others.

Contribution: In this paper, we propose a self-stabilizing clustering protocol which takes into consideration the number of nodes a clusterhead can handle, and transmission power, mobility, and battery power of the nodes. Our protocol is based on local properties. Our protocol guarantees that network nodes are partitioned into clusters: each cluster has at most SizeBound nodes. Thus, our protocol achieves load balancing by guarantee a threshold (SizeBound) on the number of nodes that a clusterhead handle. Therefore, none of the clusterheads are overloaded at any time. The clusterheads are chosen according to their weight value: the higher weight a node gets, the better clusterhead it is. Amount of bandwidth, memory space or battery power of a processor could be used to determine weight values (the computation of weight values is out the scope of this paper). A trivial solution guaranteeing the first requirements is that every node is the head of cluster having no other member. This solution does not provide an useful hierarchical organization. Therefore, we minimize the number of clusterheads in a neighborhood. Several clusterheads are neighbor only if it is necessary: no clusterhead can join another cluster and gives up its responsibility.

Our protocol is designed for the state model. Nevertheless, it can be easily transformed into a protocol for the messagepassing model. For this purpose, each node $v$ periodically broadcasts to its neighbors a message containing its state. Based on this message, $v$ 's neighbors decide to update or not their variables. After a change in the value of $v$ 's state, node $v$ broadcasts to its neighbors its new state.

The paper is organized as follows. In section 2, the formal definition of self-stabilization is presented. The wellbalanced clustering properties is presented in the section 3 . Our protocol is described in section 4. The protocol proof is presented in section 5 . We conclude by a discussion about the convergence time in section 6 .

\section{Model}

We model a distributed system by an undirected graph $G=(V, E)$ in which $V$ is the set of nodes and $E$ is the set of edges. There is an edge $(u, v) \in E$ if and only if $u$ and $v$ can directly communicate ( $u$ and $v$ are said neighbors). The set of neighbors of a node $v \in V$ will be denoted by $N_{v}$. At node $v$ in the network is assigned an unique identifier $(I D)$. For simplicity, here we identify each node with its $I D$ and we denote both with $v$. We assume the locally shared memory model of communication. Thus, each node $v$ has a finite set of local variables such that the variables at a node $v$ can be read by $v$ and any neighbors of $v$, but can be only modified by $v$.

The code of each node $v$ consists of a finite set of rule. A rule is a guarded statement of the form Rule $_{i}:$ Guard $_{i} \rightarrow$ Action $_{i}$, where Guard $_{i}$ is a boolean predicate involving the local variables of $v$ and the local variables of its neighbors, and Action $_{i}$ is a program that modify the local variables of $v$. Action $i$ is executed by node $v$ only if the Guard $_{i}$ is satisfied, in which case we say the node $v$ is enabled.

The state of a node is defined by the values of its local variables. A configuration of a distributed system $G$ is an instance of the node states. In a terminal configuration, no node is enabled.

A computation e of a system $G$ is a sequence of configurations $c_{1}, c_{2}, \ldots$ such that for $i=1,2, \ldots$, the configuration $c_{i+1}$ is reached from $c_{i}$ by a single step of one or several enabled nodes. The nodes execute their programs - code asynchronously. Therefore, the subset of enabled nodes that do 
simultaneously their action during a computation step, is arbitrary chosen. A computation is fair if any node in $G$ that is continuously enabled along a computation, will eventually perform an action. In this paper, we study only fair computation. A computation is maximal if it reaches terminal configuration or it is infinite. Let $\mathcal{C}$ be the set of possible configurations and $\mathcal{E}$ be the set of all possible computations of a system $G$. The set of computations of $G$ starting with the particular initial configuration $c \in \mathcal{C}$ will be denoted $\mathcal{E}_{c}$. The set of computations of $\mathcal{E}$ whose initial configurations are all elements of $B \in \mathcal{C}$ is denoted as $\mathcal{E}_{B}$.

In this paper, we use the notion attractor [13] to define selfstabilization.

Definition 1 (Attractor). Let $B_{3}$ and $B_{2}$ be subsets of $\mathcal{C}$. Then $B_{3}$ is an attractor from $B_{2}$ if and only if:

\section{Convergence -}

$$
\forall e \in \mathcal{E}_{B_{2}},\left(e=c_{1}, c_{2}, \ldots\right), \exists i \geq 1: c_{i} \in B_{3} .
$$

Closure - $\forall e \in \mathcal{E}_{B_{3}},\left(e=c_{1}, c_{2}, \ldots\right), \forall i \geq 1, c_{i} \in B_{3}$.

Observation 1 Let $B_{1}, B_{2}$ and $B_{3}$ be subsets of $\mathcal{C}$. If $B_{3}$ is an attractor from $B_{2}$ and if $B_{2}$ is an attractor from $B_{1}$ then $B_{3}$ is an attractor from $B_{1}$.

The set of configurations matching the problem specification is called the set of legitimate configurations, denoted as $\mathcal{L}$. $\mathcal{C} \backslash \mathcal{L}$ denotes the set of illegitimate configurations.

Definition 2 (Self-stabilization). A distributed system $S$ stabilizing to $\mathcal{S P}$ - a predicate on configurations - if and only if there exists a non-empty set $\mathcal{L} \subseteq \mathcal{C}$ such that the following conditions hold:

1. $\mathcal{L}$ is an attractor from $\mathcal{C}$.

2. $\forall c \in \mathcal{L}$, c satisfies the predicate $\mathcal{S P}$.

\section{Well-balanced Clustering for network}

We consider weight-based networks, i.e., a weight $w_{v}$ is assigned to each node $v \in V$ of the network. In ad hoc sensor networks, amount of bandwidth, memory space or battery power of a processor could be used to determine weight values. The choice of the clusterheads will be based on the weight associated to each node: the higher the weight of a node, the better this node is suitable to be a clusterhead. The nodes having an unique identifier $(I D)$, one may replace the weight variable by the uplet (weight, $I D$ ), to ensure that nodes have distinct weight values. Therefore, without loss of generality, we assume that each node has a different weight.

Clustering means partitioning its nodes into clusters, each one with a clusterhead and (possibly) some ordinary nodes. In order to have well balanced clusters, the following wellbalanced clustering properties have to be satisfied:
1. Every ordinary node always affiliates with one clusterhead of its neighborhood which has higher weight than its weight (affiliation condition).

2. There is at most SizeBound nodes in a cluster (size condition).

3. If a clusterhead $v$ has a neighboring clusterhead $u$ such that $w_{u}>w_{v}$ then the size of $u$ 's cluster is SizeBound (clusterhead neighboring condition).

$\overline{\text { Protocol } 1 \text { : definition of Constants, Variables and Macros }}$ on $v$

\section{Constants}

$w_{v}: \mathbb{N}$; - is the weight of node $v$

SizeBound: $\mathbb{N}$; - is the upper bound on a cluster size

\section{Variables of node $v$}

$C h_{v}$ : boolean; - indicate if $v$ is or is not a clusterhead.

Head $_{v}: I D s$; - is the clusterhead of $v$.

$C D_{v}:\{I D s\}$; - is the list of nodes that can choose $v$ as a their clusterhead. In the case where $v$ is an ordinary node, this list should be empty.

$S_{v}: \mathbb{N} ;$ - is the size of $v$ 's cluster. If $v$ is an ordinary node then $S_{v}$ should be 0 .

\section{Macros}

$v$ 's neighbors could be clusterheads of $v$ :

$$
\begin{gathered}
N_{v}^{+}:=\left\{z \in N_{v}: v \in C D_{z} \wedge C h_{z}=T \wedge\right. \\
\left.w_{z}>w_{\text {Head }_{v}} \wedge w_{z}>w_{v}\right\}
\end{gathered}
$$

The size of $v$ 's cluster :

$$
\text { Size }_{v}:=\mid\left\{z \in N_{v}: \text {Head}_{z}=v\right\} \mid
$$

Computation of $C D 2_{v}$ :

begin

$C D 0_{v}:=\left\{z \in N_{v}: w_{\text {Head }_{z}}<w_{v} \wedge w_{z}<w_{v}\right\}$

if $\left|C D 0_{v}\right| \leq S i z e$ Bound - Size $_{v}$ then

$C D 1_{v}:=C D 0_{v}$;

else $C D 1_{v}$ contains the SizeBound - Size smallest members of $C D 0_{v} ; \mathbf{f i}$

if $C D_{v} \subseteq C D 1_{v} \cup\left\{w \in N_{v}: \mathrm{Head}_{w}=v\right\}$

then $C D 2_{v}:=C D 1_{v}$;

else $C D 2_{v}:=\emptyset ; \mathbf{f i}$

end

The first requirement ensures that each ordinary node has direct access to its clusterhead (the head of the cluster to which it belongs), allowing fast intra and inter cluster communications. The first requirement also guarantees that each ordinary node affiliates with a suitable cluster (i.e., a cluster whose the head has a larger weight than its weight). The cluster management workload is proportional to the cluster size. Thus, the second requirement guaran- 
tees a clusterhead will be not overburden by the management workload of its cluster : each cluster has at most SizeBound members. The third requirement limits the number of clusters. A node stays clusterhead only if cannot join an existing cluster: in its neighborhood, all suitable clusters are full (i.e., they have SizeBound members).

\section{Self-stabilizing protocol building bounded size clusters}

The code of the "self-stabilizing construction of bounded size clusters" is presented in Protocol 2; the constants, the variables, and macros are defined in Protocol 1.

\section{Notation 1}

Cluster $_{v}$ is the set of nodes belonging to the v's cluster (having chosen $v$ as their clusterhead) : Cluster $v:=\{z \in$ $\left.N_{v}: \mathrm{Head}_{z}=v\right\}$. The safety predicate is defined as: $P_{s}(v) \equiv \mid C D_{v} \cup$ Cluster $_{v} \mid \leq$ SizeBound.

Our algorithm is a distributed algorithm requiring only communication between neighbors. Thus, a node gets partial information about the network state. To obtain a complete knowledge of the network state requires communication, memory space, and time that are proportional to the network size. Moreover, this knowledge will be not accurate in highly dynamic network, or large scale network where topology changes happen very often. Therefore, centralized algorithms (i.e., algorithms requiring a complete knowledge of the network) are not suitable for dynamic and large scale networks, even if they build very efficient clustering structures, as the algorithms proposed in [1].

The main idea of the algorithm is that a node becomes and stays clusterhead only when it cannot join one of its neighbor cluster without violating the well-balanced clustering properties.

To prevent that the size condition is violated, a node $u$ cannot freely join a cluster : $u$ needs to have the permission of the clusterhead. More precisely, only the nodes belonging to the set $C D_{v}$ may join $v$ 's cluster. The goal of this mechanism is to enforce the size condition. To achieve that goal, any clusterhead $v$ will verify a property stronger than the size condition, the safety predicate (defined above). A cluster whose the head satisfying the safety predicate verifies the size condition after any computation step. A contrario, a cluster whose the head $v$ does not satisfy the safety predicate, may not verify the size condition, after a specific computation step (i.e., all nodes belonging to $C D_{v}$ join $v$ 's cluster). Therefore, we have designed our protocol is such a way that the safety predicate is eventually always satisfied by all clusterheads.

For each clusterhead $v$, the macro $C D 2_{v}$ is used to set $C D_{v}$ value. $C D 0_{v}$ is the set of $v$ 's neighbors wanting to join

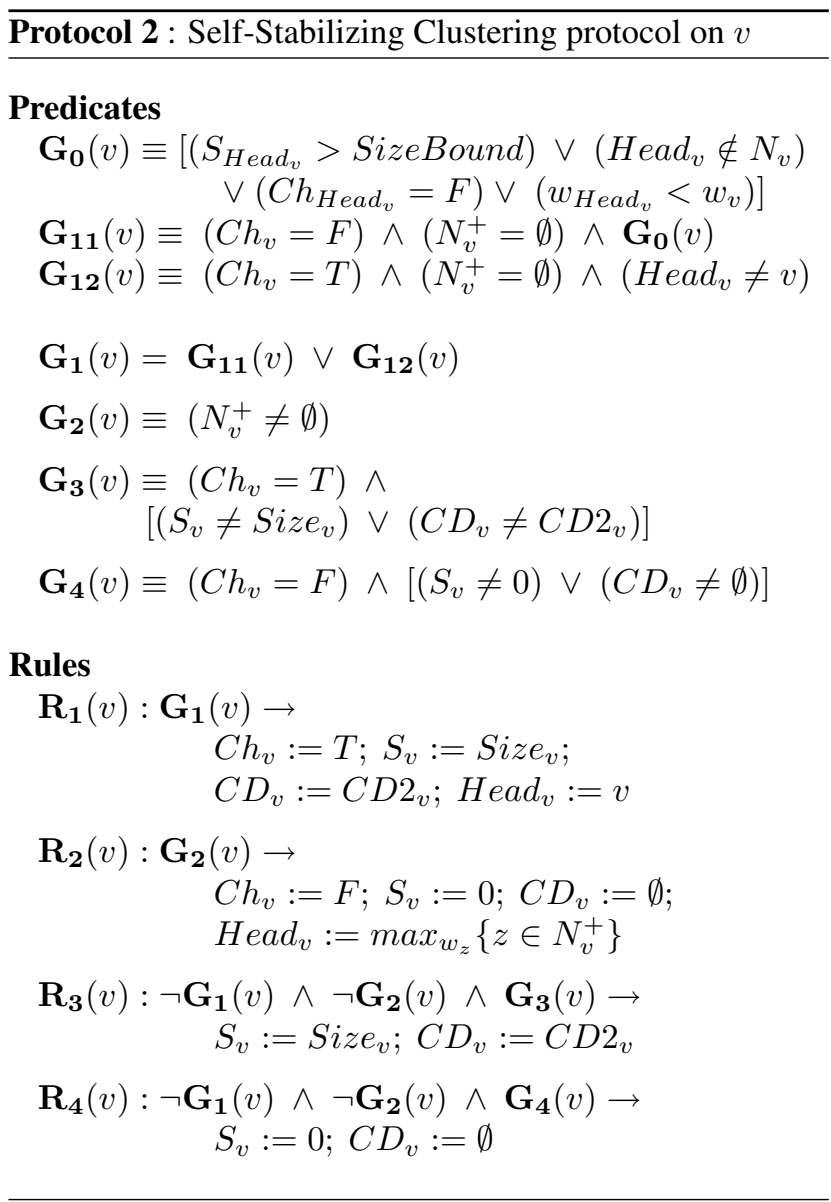

: Clusterhead node

O: Ordinary node

SizeBound $=3$

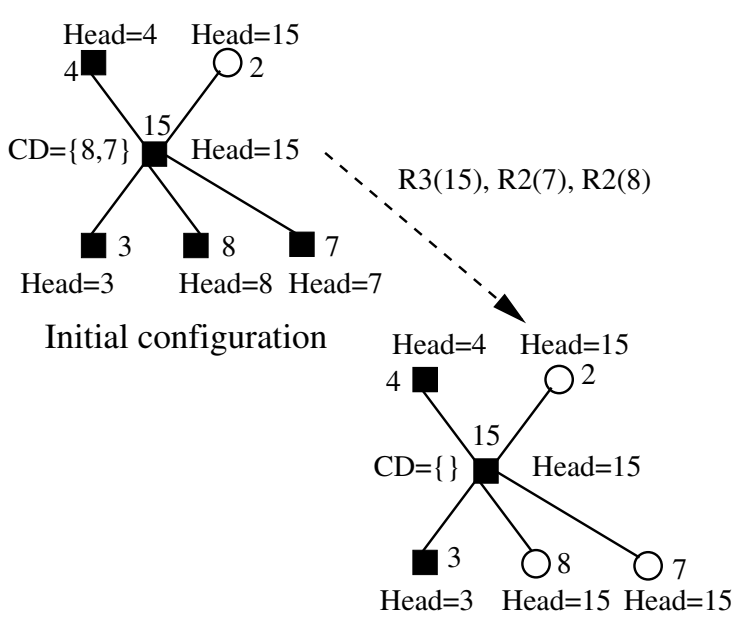

Figure 1. Illustration of $C D$ value computation 
the $v$ 's cluster (i.e., their clusterhead has a smaller weight than $v^{\prime}$ weight). Only a subset of $C D 0_{v}$ is selectionned to be in $C D 2_{v}$. This subset of $C D 0_{v}$ is computed to ensure that the safety predicate would be satisfied by $v$ after any computation step from the current configuration. Let us illustrate the computation of $C D 2$ by an example. In the initial configuration of the Figure 1 , the safety predicate is satisfied (i.e., every node $v$ satisfies the predicate $P_{s}(v)$ ). In this configuration, the node having the biggest weight is node 15 . All its neighbors want to join its cluster $C D 0_{15}=N_{15}-$ Cluster $_{15}=\{3,4,7,8\}$. Node 15 can allow only two nodes to join its cluster (because SizeBound $=3$ ). The two selected nodes are the nodes of $C D 0_{15}$ having the two-smallest weight. Therefore $C D 1_{15}=\{3,4\}$. In the Figure 1, during the first computation step, all nodes of $C D 0_{15}$ join Cluster Cl5 $_{15}$. At the end of this computation step, Cluster $_{15}=\{2,7,8\}$. If $C D_{15}$ would have the value $C D 1_{15}=\{3,4\}$. After this step, $\mid C D_{15} \cup$ Cluster $_{15} \mid$ could have the value $5=|\{2,3,4,7,8\}|$, the safety predicate would not be satisfied: after another computation step, the 15 's cluster could have 5 members. Therefore, $C D_{15}$ is set to $\emptyset$, and not to $C D 1_{15}$.

The macro $N_{v}^{+}$helps us to guarantee the clusterhead neighboring condition. The set $N_{v}^{+}$is not empty, if the clusterhead $v$ does not verify the clusterhead neighboring condition. In this case, $v$ is enabled ; it has to join an existing cluster in its neighborhood. $N_{v}^{+}$contains the list of $v$ ' neighbors that are better clusterheads for $v$ than its current one. Node $v$ will choose the node of $N_{v}^{+}$having the biggest weight as its clusterhead (rule $R_{2}$ ). The $N_{v}^{+}$set contains neighbors of $v$ verifying the following properties (1) they are clusterhead, (2) their weight is bigger than the weight of the current clusterhead of $v$, (3) their weight is bigger than the weight of $v$, and (4) they accept that $v$ joins their cluster (i.e., $v$ belongs to their $C D$ set).

We split the possible cases where a node $v$ has to change its local variables according to the following mutually exclusive cases:

Case 1. $v$ has to become a clusterhead (rule $R_{1}$ ). In $v$ 's neighborhood, there is not suitable clusterhead (i.e., $N_{v}^{+}$ is empty) and it belongs to a cluster having more than SizeBound nodes or $v$ does not satisfy the Affiliation condition. In this case, $G_{11}(v)$ is satisfied.

Case 2. $v$ has to changed of cluster (rule $R_{2}$ ). The set of $N_{v}^{+}$is not empty: $v$ has in its neighborhood a more suitable clusterhead than its current one. In this case, $G_{2}(v)$ is satisfied.

Case 3. $v$ has to change some local variable values ( $S$ or $C D$ value) without changing of cluster (it will stay clusterhead or ordinary). If $v$ is a clusterhead then $G_{3}(v)$ or $G_{12}(v)$ is satisfied. If $v$ is an ordinary node then $G_{4}(v)$ is satisfied.
: Clusterhead node

O: Ordinary node

SizeBound=3
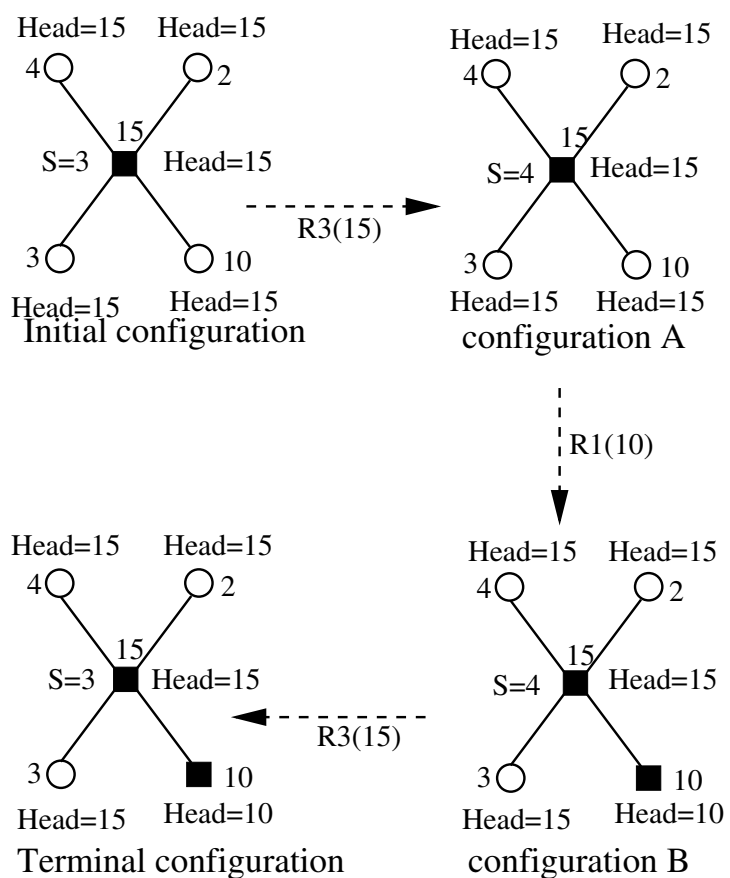

Figure 2. Illustration of Self-stabilizing construction of bounded clusters

Protocol 1 is illustrated in Figure 2, in this example SizeBound $=3$. Initially, there is one cluster having 4 members (the size condition is not verified). The node 10 will quit this cluster, and will form a new cluster (action $R_{1}$ ), once the only clusterhead (node 15 ) has updated its $S$ variable (action $R_{3}$ ).

\section{Proof of self-stabilization}

In subsection 5.1, we prove that the safety predicate holds continuously till the protocol converges to a legitimate configuration, once a configuration reaching this predicate is reached. Such an example is given in figure 3. Initially all clusters have less than 3 members, this property is verified along any computation reaching a legitimate configuration.

A legitimate configuration is a terminal configuration where every node $v$ satisfies $P_{s}(v)$ and $C D_{v}=\emptyset$. In subsection 5.2 , we prove that along any fair computation, a legitimate configuration is reached from any configuration. In subsection 5.3, we establish that in a legitimate configuration, the well-balanced clustering properties are verfied. 


\subsection{Safety}

Definition 3 Let $A_{2}$ be the set of safe configurations defined by $\left\{\mathcal{C} \mid \forall v: P_{s}(v)\right.$ is satisfied $\}$.

Notation 2 Let $c$ be a configuration. We denote $C D_{v}(c)$ the value of the $C D$ variable of the node $v$ in $c$. We denote Cluster $_{v}(c)$ the v's cluster in $c$.

Observation 2 Assume that we have a computation step $c_{1} \stackrel{c s}{\rightarrow} c_{2}$. According to the macro $N^{+}$and to the rule $R_{2}$, Cluster $_{v}\left(c_{2}\right) \subseteq\left(\right.$ Cluster $\left._{v}\left(c_{1}\right) \cup C D_{v}\left(c_{1}\right)\right)$.

Assume that $v$ updates $C D_{v}$ during the computation step cs. According to the macro $C D 2_{v}$,

- $C D_{v}\left(c_{2}\right)=\emptyset$ or

$\mid C D_{v}\left(c_{2}\right) \cup$ Cluster $_{v}\left(c_{1}\right) \mid \leq$ SizeBound

- $C D 1_{v}\left(c_{1}\right) \cap$ Cluster $_{v}\left(c_{1}\right)=\emptyset$.

Lemma $1 A_{2}$ is closed.

Proof: Assume that: (1) we have a configuration $c_{1}$ in which $P_{s}(v)$ holds and (2) we have a computation step $c_{1} \stackrel{c s}{\rightarrow} c_{2}$. We will prove that $P_{s}(v)$ holds in $c_{2}$.

In any case $\left(C D_{v}\left(c_{2}\right)=C D_{v}\left(c_{1}\right), C D_{v}\left(c_{2}\right)=\right.$ $\emptyset, \quad$ or $\left.C D_{v}\left(c_{2}\right)=C D 2_{v}\left(c_{1}\right)\right)$, we have $\mid C D_{v}\left(c_{1}\right) \cup$ Cluster $_{v}\left(c_{1}\right) \mid \leq$ SizeBound and $\mid C D_{v}\left(c_{2}\right) \cup$ Cluster $_{v}\left(c_{1}\right) \mid \leq$ SizeBound.

Case 1: Cluster $_{v}\left(c_{2}\right) \subseteq$ Cluster $_{v}\left(c_{1}\right) . P_{s}(v)$ is satisfied in $c_{2}$.

Case 2: Cluster $_{v}\left(c_{2}\right) \subseteq\left\{u_{1}, . . u_{m}\right\} \cup$ Cluster $_{v}\left(c_{1}\right)$. According to the observation 2, we have $\left\{u_{1}, . . u_{m}\right\} \subset$ $C D_{v}\left(c_{1}\right)$.

If $C D_{v}\left(c_{2}\right)=C D_{v}\left(c_{1}\right)$ or $C D_{v}\left(c_{2}\right)=\emptyset$ then $\mid C D_{v}\left(c_{2}\right) \cup$ Cluster $_{v}\left(c_{2}\right)|\leq| C D_{v}\left(c_{1}\right) \cup$ $\left\{u_{1}, . . u_{m}\right\} \cup$ Cluster $_{v}\left(c_{1}\right)|\leq| C D_{v}\left(c_{1}\right) \cup$ Cluster $_{v}\left(c_{1}\right) \mid$ $\leq$ SizeBound. $P_{s}(v)$ is satisfied in $c_{2}$.

If $C D_{v}\left(c_{2}\right)=C D 2_{v}\left(c_{1}\right)$ then (according to the $C D 2_{v}$ definition) $C D_{v}\left(c_{1}\right) \subset\left(C D_{v}\left(c_{2}\right) \cup\right.$ Cluster $\left._{v}\left(c_{1}\right)\right)$.

Thus $\mid C D_{v}\left(c_{2}\right) \quad \cup \quad$ Cluster $_{v}\left(c_{2}\right) \mid=$ $\mid C D_{v}\left(c_{2}\right) \cup\left\{u_{1}, \quad . . u_{m}\right\} \cup$ Cluster $_{v}\left(c_{1}\right) \mid \leq$ $\mid C D_{v}\left(c_{2}\right) \cup$ Cluster $_{v}\left(c_{1}\right) \mid \leq$ SizeBound.

$P_{s}(v)$ is satisfied in $c_{2}$.

\subsection{Convergence}

In this subsection, some of the proofs are omitted dee to lack of space, they can be found in [10].

Lemma $2 P_{g} \equiv\left(G_{12}(v)=F\right) \wedge\left(G_{4}(v)=F\right)$.

$A_{4}=A_{2} \cap\left\{c \in \mathcal{C} \mid \forall v:\right.$ Head $_{v} \in N_{v} \cup\{v\}$ and $\mid$ Cluster $_{v} \mid \leq$ SizeBound and $P_{g}(v)$ is satisfied $\}$ is an attractor from $\mathcal{C}$.
: Clusterhead node

: Ordinary node

Sizebound $=3$

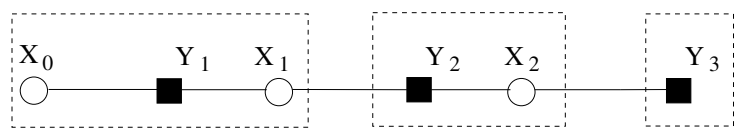

(a) Initial configuration

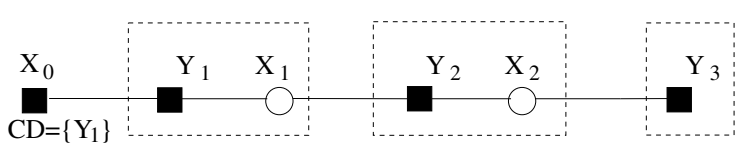

(b) Configuration after the 1st computation step

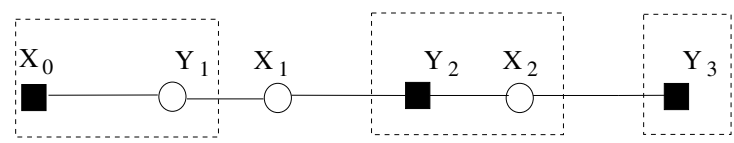

(c) Configuration after the 2nd computation step

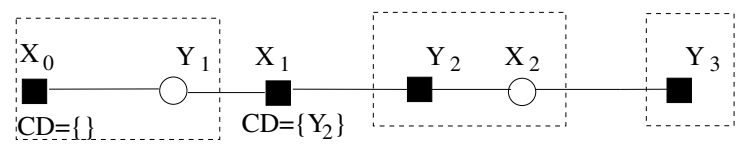

(d) Configuration after the 3rd computation step

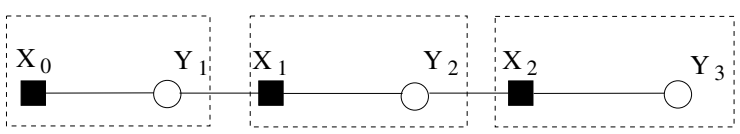

Terminal configuration

Figure 3. Stabilization time.

The convergence is done in step. At the end of the $i^{t h}$ step the configuration set $L "{ }_{i}$ is reached: all nodes of $S e t_{i}$ has chosen forever their clusterhead. We define $S e t_{i}$ and $L^{\prime \prime}{ }_{i}$ (for any value of $i$ ) as follows.

\section{Notation 3}

$L{ }_{0}=A_{4}$ and $S e t_{0}=\emptyset$.

$V_{i}$ is the set of nodes that do not belong to Set ${ }_{i}: V_{i}=$ $V-$ Set $_{i}$.

$v h_{i}$ is the node with the highest weight in $V_{i}$.

$L_{i+1}=L{ }_{i} \cap\left\{c \in \mathcal{C} \mid C h_{v h_{i}}=T\right\}$

SizeBound $_{i}$ is the value min (SizeBound, $\left.\left|N_{v h_{i}} \cap V_{i}\right|\right)$.

$L_{i+1}^{\prime}=L_{i+1} \cap\left\{c \in \mathcal{C}||\right.$ Cluster $_{v h_{i}} \mid=$ SizeBound $\left._{i}\right\}$.

Set $_{i+1}=$ Set $_{i} \cup\left\{v h_{i}\right\} \cup$ Cluster $_{v h_{i}}$.

$L{ }^{\prime}{ }_{i+1}=L_{i+1}^{\prime} \cap\left\{c \in \mathcal{C} \mid \forall v \in \operatorname{Set}_{i+1}: C D_{v}=\emptyset\right\}$. 
The convergence steps are illustrated in figure 3 where the weight of nodes are ordered as the following: $X_{i-1}>Y_{i}>$ $X_{i}>Y_{i+1}$. Initially $A_{4}$ is reached. After the first computation step, $L_{1}$ is reached (the node having the largest weight, $X_{0}$, is a clusterhead). After the second computation step $L_{1}^{\prime}$ is reached $\left(\right.$ Cluster $X_{X_{0}}=\left\{Y_{1}\right\}=N_{X_{0}}$ ). After the third computation step, $L_{2}$ is reached, $X_{1}$, the node having the largest weight of $V-\left\{X_{0}, Y_{1}\right\}$, is a clusterhead. After the fourth computation step, $L_{2}^{\prime}$ is reached (Cluster $\left._{X_{1}}=\left\{Y_{2}\right\}=N_{X_{1}}-\left\{X_{0}, Y_{1}\right\}\right)$.

Observation 3 Let $v$ be a node of $V_{i}$. We have, by definition of $V_{i}$, Head $_{v} \notin$ Set $_{i}$. If Set S $_{i} \neq V$ then $\left(\right.$ Set $_{i} \subset$ Set $\left._{i+1}\right)$ and $\left(\right.$ Set $_{i} \neq$ Set $\left._{i+1}\right)$.

At each step, Set $t_{i}$ increases up to contain all nodes. Once $S_{e} t_{i}=V$, we will prove that a legitimate configuration is reached.

Lemma 3 For any value of $i, L_{i+1}$ is an attractor from $\mathcal{C}$, assuming that $L "{ }_{i}$ is an attractor from $\mathcal{C}$.

Proof: $v h_{i}$ is the node with the biggest weight in $V_{i}$. Let $z$ be in $N_{v h_{i}}$. If $z \in S e t_{i}$, we have $C D_{z}=\emptyset$ (see definition of $L{ }_{0}{ }_{0}$ or $\left.L "{ }_{i+1}\right)$. So $N_{v h_{i}}^{+}$is empty: $v h_{i}$ never executes $R_{2}\left(v h_{i}\right)$.

According to the observation 3, we have $\mathrm{Head}_{v h_{i}} \in V_{i}$, thus by definition of $v h_{i}, w_{\text {Head }_{v h_{i}}} \leq w_{v h_{i}}$.

If $v h_{i}$ is not a clusterhead then $G_{1}\left(v h_{i}\right)$ is satisfied because $w_{\text {Head }_{v h_{i}}}<w_{v h_{i}}$. As all computations are fair, $v h_{i}$ eventually performs $R_{1}\left(v h_{i}\right)$. After that $C h_{v h_{i}}=T$ and $\operatorname{Head}_{v h_{i}}=v h_{i}$, forever.

Lemma 4 Let $c_{1}$ be a configuration of $L_{i+1}$. Let cs be a computation step from $c_{1}: c_{1} \stackrel{c s}{\longrightarrow} c_{2}$. We have Cluster $_{v h_{i}}\left(c_{1}\right) \subseteq$ Cluster $_{v h_{i}}\left(c_{2}\right)$.

\section{Proof:}

Let $u$ be a node of Cluster $v h_{i}$ (i.e., Head Hevh $_{i}$ ). In $L_{i+1}$, a neighbor $z$ of $u$ such that $w_{z}>w_{v h_{i}}$ is in $\operatorname{Set}_{i}$ : $C D_{z}=\emptyset$. Thus $z \notin N_{u}^{+}$; we conclude that $N_{u}^{+}$is empty forever. Thus, $G_{2}(u)$ is never verified. In $L_{i+1}, G_{1}(u)$ is not verified. We conclude that the node $u$ stays in the Cluster of $v h_{i}$ forever.

Lemma 5 Let $c_{1}$ be a configuration of $L_{i+1}$. Let $c s$ be a computation step from $c_{1}$ : $c_{1} \stackrel{c s}{\longrightarrow} c_{2}$. We have $C D 1_{v h_{i}}\left(c_{2}\right) \neq C D 1_{v h_{i}}\left(c_{1}\right)$ if only if Cluster ${ }_{v h_{i}}\left(c_{1}\right) \neq$ Cluster $_{v h_{i}}\left(c_{2}\right)$.

Lemma 6 For any value of $i, L_{i+1}^{\prime}$ is an attractor from $\mathcal{C}$ assuming that $L_{i+1}$ is an attractor from $\mathcal{C}$.
Proof: Once $L_{i+1}$ is reached, only the nodes of $V_{i}$ may be in the Cluster of $v h_{i}$, therefore, the size of Cluster $v h_{i}$ is bounded by SizeBound . $_{\text {. }}$

As no node can quit Cluster $_{v h_{i}}$ (lemma 4), Cluster $_{v h_{i}}$ will eventually stay identical forever. According lemma 5, $C D 1_{v h_{i}}$ will eventually stay identical forever.

Once $C D 1_{v h_{i}}$ is set up, if, $C D D_{v h_{i}}=C D 1_{v h_{i}}, R_{3}\left(v h_{i}\right)$ is never enabled (i.e., $C D_{v h_{i}}$ stays always equal to $C D 1_{v h_{i}}$ ). Once, $C D 1_{v h_{i}}$ is set up, if $C D_{v h_{i}} \neq C D 1_{v h_{i}}$ then $R_{3}\left(v h_{i}\right)$ is enabled forever. By fairness, $R_{3}\left(v h_{i}\right)$ action will be eventually performed. After at most two $R_{3}\left(v h_{i}\right)$ actions, we have $C D_{v h_{i}}=C D 1_{v h_{i}}$. We conclude that any computation has a suffix where $C D_{v h_{i}}$ stay equal to $C D 1_{v h_{i}}$. In this suffix, the size of $C D D_{v h_{i}}$ is equal to SizeBound $_{i}-\mid$ Cluster $_{v h_{i}} \mid$

Assume that size of Cluster $_{v h_{i}}$ is forever smaller than SizeBound $_{i}$. In that case, the computation has a suffix where a node $u$ will stay forever in the set $C D_{v h_{i}}$. By definition of $v h_{i}$ and $V_{i}$, we have $w_{\text {Head }_{u}}<w_{v h_{i}}$ and $w_{u}<w_{v h_{i}}$, thus $v h_{i} \in N_{u}^{+}$. Any neighbor $z$ of $u$ such that $w_{z}>w_{v h_{i}}$ is in $\operatorname{Set}_{i}$. Thus $C D_{z}=\emptyset$. Therefore $v h_{i}$ is the node of $N_{u}^{+}$having the biggest weight. $R_{2}(u)$ is enabled forever. By fairness, $R_{2}(u)$ action will be eventually performed: $u$ will choose $v h_{i}$ as its clusterhead: Cluster $_{v h_{i}}$ is modified. There is a contradiction.

Lemma 7 For any value of $i, L "{ }_{i+1}$ is an attractor from $\mathcal{C}$, assuming that $L_{i+1}^{\prime}$ is an attractor from $\mathcal{C}$.

Proof: Let $v$ be a node of $S e t_{i+1}$ that does not belong to $S e t_{i}$. If $v$ is an ordinary node, $C D_{v}=\emptyset$ because $A_{3}$ is a subset of $L "{ }_{i+1}$. If $v$ is a clusterhead then $v=v h_{i}$. By definition of $L_{i+1}^{\prime}, \forall u \in N_{v}: w_{\text {Head }_{u}} \geq w_{v}$ or $\mid$ Cluster $_{v} \mid=$ SizeBound. Thus $C D 2_{v}=\emptyset$, in $L_{i+1}^{\prime}$. If $C D_{v} \neq \emptyset$, then $R_{3}(v)$ is enabled forever. Once the rule is executed, $C D_{v}=\emptyset$ holds.

Theorem 1 Let $A_{5}$ a configurations set defined by $A_{5}=$ $A_{4} \cap\left\{c \in \mathcal{C} \mid \forall v: C D_{v}=\emptyset\right\}$. The system eventually reaches a terminal configuration of $A_{5}$.

Proof: According to the Observation 3, Set $_{i} \subset$ Set $_{i+1}$. Thus, there exists $j$ such that $\operatorname{Set}_{j}=V$.

$L "{ }_{j}$ is an attractor because $L{ }_{0}, L_{i}, L_{i}^{\prime}$, and $L{ }^{\prime}{ }_{i}$ are attractors for any value of $1 \geq i \leq j$. In $L "{ }_{j}$, the rule $R_{1}$, the rule $R_{2}$, and the rule $R_{4}$ are not enabled on any node. Only the rule $R_{3}$ may be enabled forever, on a node $v$. By fairness, $v$ will execute $R_{3}(v)$, then $v$ is never enabled. We conclude that a terminal configuration of $L_{i}^{\prime \prime}$ will be reached. Any configuration of $L_{i}^{\prime \prime}$ belong to $A_{5}$. 


\subsection{Correctness}

Theorem 2 Once a terminal configuration of $A_{5}$ is reached, the well-balanced clustering properties are satisfied.

Proof: In a terminal configuration of $A_{5}$, for every node $z$, we have $G_{i}(z)=F: i=1 . .4$ and $C D_{z}=\emptyset$ (see theorem 1).

Case 1. $z$ is an ordinary node. $G_{11}(z)=F$ implies $\left(\right.$ Head $\left._{z} \in N_{v}\right) \wedge\left(C_{\text {Head }_{z}}=T\right)$ and $\left(w_{\text {Head }_{z}}>w_{z}\right)$. Thus, $z$ satisfies affiliation condition.

Case 2. $z$ is a clusterhead node. Following Lemma 2, in a terminal configuration $S_{z} \leq$ SizeBound, thus, the size condition is satisfied.

Let $v$ be a clusterhead, neighbor of $z$ such that $w_{v}>w_{z}$. Notice that $C D 0_{v}$ is not empty (it contains $z$ ). $G_{3}(v)$ is not verified. Thus, $C D 2_{v}$ is equal to $C D_{v}$. We have $C D_{v}=\emptyset$, thus, $C D 2_{v}=C D 1_{v}=\emptyset$. We conclude that Size $_{v}=$ SizeBound. Thus, every clusterhead $v$ in $z$ ' neighborhood verifies the following predicate: $\left(w_{v} \leq w_{z}\right)$ or $\left(S_{v}=\right.$ SizeBound $)$. Therefore, the clusterhead neighboring condition is satisfied.

\section{Convergence times}

The stabilization time is the maximum number of computation steps needed to reach a stabilized configuration from an arbitrary initial one. Figure 3 presents a scenario to measure stabilization time, in the worst case: the initial configuration is the worst one. In this configuration (Figure 3.a), there are $(N-1) / 2$ clusters where $N$ is the network size. Each cluster $C_{i}(i>1)$ includes a clusterhead $Y_{i}$ and a ordinary node $X_{i+1}$. The weight of nodes are ordered as the following: $X_{i-1}>Y_{i}>X_{i}>Y_{i+1}$. Initially, only the node $X_{0}$ is enabled. $X_{0}$ is not a clusterhead, and its has the largest weight of the system; thus $G_{11}\left(X_{0}\right)$ is satisfied. In the first round $X_{0}$ performs the rule $R_{1}$. Now, $Y_{1}$ is enabled, because $N_{Y_{1}}^{+}=\left\{X_{0}\right\}$; the other nodes are still not enabled. Thus, during the second round, the node $Y_{1}$ performs the rule $R_{2}$ to join $X_{0}$ 's cluster. Then, $X_{1}$ is enabled; the $G_{11}\left(X_{1}\right)$ predicate is satisfied because $C l_{\text {Head }_{X 1}}=F$. In the third round, $X_{1}$ performs the rule $R_{1}$, and so one. All nodes will update their status; during a computation step, only one node changes its status.

The stabilization time is $O(N)$.

\section{References}

[1] B. Awerbuch and D. Peleg. Sparse partitions (extended abstract). In the 31st Annual IEEE Symposium on Foundations of Computer Science (FOCS90), pages 503-513, 1990.
[2] S. Banerjee and S. Khuller. A clustering scheme for hierarchical control in multi-hop wireless networks. In the 20th Conference of the IEEE Communications Society (INFOCOM'01), pages 1028-1037, 2001.

[3] S. Basagni. Distributed and mobility-adaptive clustering for multimedia support in multi-hop wireless networks. In the IEEE 50th International Vehicular Technology Conference (VTC'99), pages 889-893, 1999.

[4] S. Basagni. Distributed clustering for ad hoc networks. In the 1999 International Symposium on Parallel Architectures, Algorithms, and Networks (ISPAN'99), pages 310315, 1999.

[5] D. Bein, A. K. Datta, C. R. Jagganagari, and V. Villain. A self-stabilizing link-cluster algorithm in mobile ad hoc networks. In the 8th International Symposium on Parallel Architectures, Algorithms and Networks (ISPAN'05), pages 436-441, 2005.

[6] E. M. Belding-Royer. Multi-level hierarchies for scalable ad hoc routing. Wireless Networks, 9(5):461-478, 2003.

[7] M. Chatterjee, S. Das, and D. Turgut. WCA: A weighted clustering algorithm for mobile ad hoc networks. Journal of Cluster Computing, Special issue on Mobile Ad hoc Networking, 5(2):193-204, 2002.

[8] E. Dijkstra. Self stabilizing systems in spite of distributed control. Communications of the Association of the Computing Machinery, 17:643-644, 1974.

[9] Y. Fernandess and D. Malkhi. K-clustering in wireless ad hoc networks. In the 2nd ACM international workshop on Principles of mobile computing (POMC'02), pages 31-37, 2002.

[10] C. Johnen and L. Nguyen. Self-stabilizing bounded size clustering algorithm. Technical Report 1464, L.R.I, 2006.

[11] C. Johnen and L. H. Nguyen. Robust self-stabilizing clustering algorithm. In the 10th International Conference On Principles Of Distributed Systems (OPODIS'06), Springer LNCS 4305, pages 408-422, 2006.

[12] C. Johnen and L. H. Nguyen. Self-stabilizing weight-based clustering algorithm for ad hoc sensor networks. In the 2nd International Workshop on Algorithmic Aspects of Wireless Sensor Networks (ALGOSENSORS'06), Springer LNCS 4240, pages 83-94, 2006.

[13] C. Johnen and S. Tixeuil. Route preserving stabilization. In the 6th International Symposium on Self-stabilizing System (SSS'03), Springer LNCS 2704, pages 184-198, 2003.

[14] H. Kakugawa and T. Masuzawa. A self-stabilizing minimal dominating set algorithm with safe convergence. In the 8th IPDPS Workshop on Advances in Parallel and Distributed Computational Models (APDCM'06), 2006.

[15] N. Mitton, A. Busson, and E. Fleury. Self-organization in large scale ad hoc networks. In the 3rd Annual Mediterranean Ad Hoc Networking Workshop (MED-HOCNET'04), June 2004.

[16] N. Mitton, E. Fleury, I. Guérin-Lassous, and S. Tixeuil. Self-stabilization in self-organized multihop wireless networks. In the 25th IEEE International Conference on Distributed Computing Systems Workshops (WWAN'05), pages 909-915, 2005.

[17] F. G. Nocetti, J. S. Gonzalez, and I. Stojmenovic. Connectivity based $k$-hop clustering in wireless networks. Telecommunication Systems, 22(1-4):205-220, 2003. 